\title{
Article \\ Control Strategies for Gait Tele-Rehabilitation System Based on Parallel Robotics
}

\author{
Antonio P. L. Bo ${ }^{1,+} \mathbb{D}$, Leslie Casas ${ }^{2}$, Gonzalo Cucho-Padin ${ }^{3,4} \mathbb{( D}$, Mitsuhiro Hayashibe ${ }^{5, \ddagger}$ and Dante Elias ${ }^{6, *(\mathbb{D})}$ \\ 1 Electrical Engineering Department, University of Brasilia, Brasilia 70910-900, Brazil; antonio.plb@uq.edu.au \\ 2 Department of Computer Aided Medical Procedures and Augmented Reality, \\ Technische Universität München, 80333 München, Germany; leslie.casas.g@gmail.com \\ 3 Department of Physics, Catholic University of America, Washington, DC 20064, USA; \\ gonzaloaugusto.cuchopadin@nasa.gov \\ 4 NASA Goddard Space Flight Center, Greenbelt, MD 20771, USA \\ 5 French Institute for Research in Computer Science and Automation (INRIA), \\ 78150 Paris, France; hayashibe@tohoku.ac.jp \\ 6 Laboratory of Research in Biomechanics and Applied Robotics, Mechanical Engineering Department, \\ Pontificia Universidad Católica del Perú (PUCP), Lima 15088, Peru \\ * Correspondence: delias@pucp.pe \\ + Current address: School of Information Technology and Electrical Engineering, \\ The University of Queensland, Brisbane, QLD 4072, Australia. \\ $\ddagger$ Current address: Department of Robotics, Graduate School of Engineering, Tohoku University, \\ Miyagi 980-8577, Japan.
}

Citation: Bo, A.P.L.; Casas, L.; Cucho-Padin, G.; Hayashibe, M.; Elias, D. Control Strategies for Gait Tele-Rehabilitation System Based on Parallel Robotics. Appl. Sci. 2021, 11, 11095. https://doi.org/10.3390/ app112311095

Academic Editors: Inaki Diaz and Jorge Juan Gil

Received: 22 October 2021

Accepted: 15 November 2021

Published: 23 November 2021

Publisher's Note: MDPI stays neutral with regard to jurisdictional claims in published maps and institutional affiliations.

Copyright: (c) 2021 by the authors. Licensee MDPI, Basel, Switzerland. This article is an open access article distributed under the terms and conditions of the Creative Commons Attribution (CC BY) license (https:// creativecommons.org/licenses/by/ $4.0 /)$.

\begin{abstract}
Among end-effector robots for lower limb rehabilitation, systems based on Stewart-Gough platforms enable independent movement of each foot in six degrees of freedom. Nevertheless, control strategies described in recent literature have not been able to fully explore the potential of such a mechatronic system. In this work, we propose two novel approaches for controlling a gait simulator based on Stewart-Gough platforms. The first strategy provides the therapist direct control of each platform using movement data measured by wearable sensors. The following scheme is designed to improve the level of engagement of the patient by enabling a limited degree of control based on trunk inclination. Both strategies are designed to facilitate future studies in tele-rehabilitation settings. Experimental results have illustrated the feasibility of both control interfaces, either in terms of system performance or user subjective evaluation. Technical capacity to deploy in tele-rehabilitation was also verified in this work.
\end{abstract}

Keywords: gait simulator; Stewart-Gough platforms; inertial sensing; tele-rehabilitation

\section{Introduction}

In recent years, special attention has been given to the development of mechatronic rehabilitation systems, which can provide high intensity and frequency of treatment for improved motor recovery [1], particularly when used in a tele-rehabilitation framework. For lower limb rehabilitation, robotic systems are now commercially available as an alternative to conventional therapy, and technological complement to Body-Weight Supported Treadmill Training (BWSTT) [2,3].

Generally, robot-assisted lower limb therapy consists of the simulation of joint or foot path, such as gait trajectories produced by non-impaired people. The resulting movement may possibly integrate other technologies, such as Functional Electrical Stimulation [4], or additional levels of control, such as the ability to assist-as-needed [5,6]. Mechanical support is often delivered to the patient using either an exoskeletal device [2], or an end-effector-based robot $[3,5]$. While exoskeleton-based systems may lessen the strain on therapists, studies suggest that patient engagement on therapy may also be reduced [7], which may indicate that the neurological pathways required for rehabilitation are not 
properly stimulated. In view of this issue, recent research has proposed new treadmill control schemes [8], as well as structurally innovative treadmills [9]. Nevertheless, in this work, we focus on end-effector systems.

End-effector-based lower limb rehabilitation systems represent an alternative to these approaches. Some of those systems were designed for BWSTT [3,5], while others have targeted ankle rehabilitation only [10,11]. To the best of our knowledge, none of these systems provide BWSTT capability and independent six degree-of-freedom (DOF) movement of each foot. A system combining such features may not only be used for gait training, but also in balance assessment and exercises, an area that has also seen development of specific methods [12] and devices [13].

This study utilizes a gait simulator system based on two Stewart-Gough platforms used for lower limb rehabilitation. A thorough description of its mechanical design and implementation is reported in [14]. We propose in this paper novel control strategies that provide the system with additional tele-rehabilitation capabilities.

In terms of control strategies, force control is often used in robot-aided rehabilitation and, more generally, in human-robot interaction. Despite the advantages of approaches based on force control, challenges exist, particularly in a tele-rehabilitation scenario [15], to apply these methods in our SGP-based system. Hence, the original version of the gait simulator employed position control, simply emulating standard gait trajectories, which remained fixed for the duration of the rehabilitation session. In order to improve overall safety (especially for use in tele-rehabilitation), provide a more intuitive therapist interface, and enable more active patient engagement, two main techniques are proposed in this paper.

Firstly, we propose a robot walking velocity modulation based on patient motion (Self-Modulation Mode, SMM), which is similar to the idea proposed in [13]. However, instead of using a mechanical structure attached to the upper limb, we have used wearable sensors on the trunk, and velocity is updated based on the patient's posture. Secondly, the platforms may also be directly controlled using the therapist's mirrored motion (Therapist Modulation Mode, TMM). In our work, we have applied a similar approach as in [16], i.e., by using an intuitive interface based on wearable sensors, the therapist may control the slave robotic system intuitively and possibly from a remote location.

Indeed, both control strategies proposed in this work may be further expanded by the use of tele-rehabilitation, in which the therapist is located remotely [17]. Within a session employing TMM, the therapist may control the system motion using his own body movement, while monitoring patient response. When the system is operated using the SMM, the therapist may remotely assess patient movement based on video and data from the wearable sensors, particularly if methods for automatic quantitative assessment are used $[18,19]$. In order to validate the tele-rehabilitation component of this system, series of simulated experiments were conducted to verify the feasibility of our technical solution.

This paper is organized as follows. Section 2 presents the gait simulator, including details on its mechanical design, low-level control, and additional development required to implement new operation modes, as well as the proposed control strategies, SMM and TMM. The method employed to validate the tele-rehabilitation component is also described in this Section. Experimental evaluation is presented in Section 3, including data from tests to validate each subsystem separately and preliminary experiments designed to evaluate the feasibility of the overall system when using the novel operation modes. These trials involved healthy subjects. These results are discussed in Section 4. Finally, we draw conclusions and discuss future works in Section 5.

\section{Materials and Methods}

\subsection{Gait Simulator}

The gait simulator employed in this work is based on the operation of two parallel manipulators capable of performing on-site walking. Its main goal is to induce lower limb movements on a user standing on his/her feet over the platform. As depicted 
in Figure 1, the platform is designed to enable independent 6-DOF movement of each foot, thus producing motions ranging from normal walking to arbitrary lower extremity movements, hence potentially contributing for the restoration of motor function in different clinical scenarios.

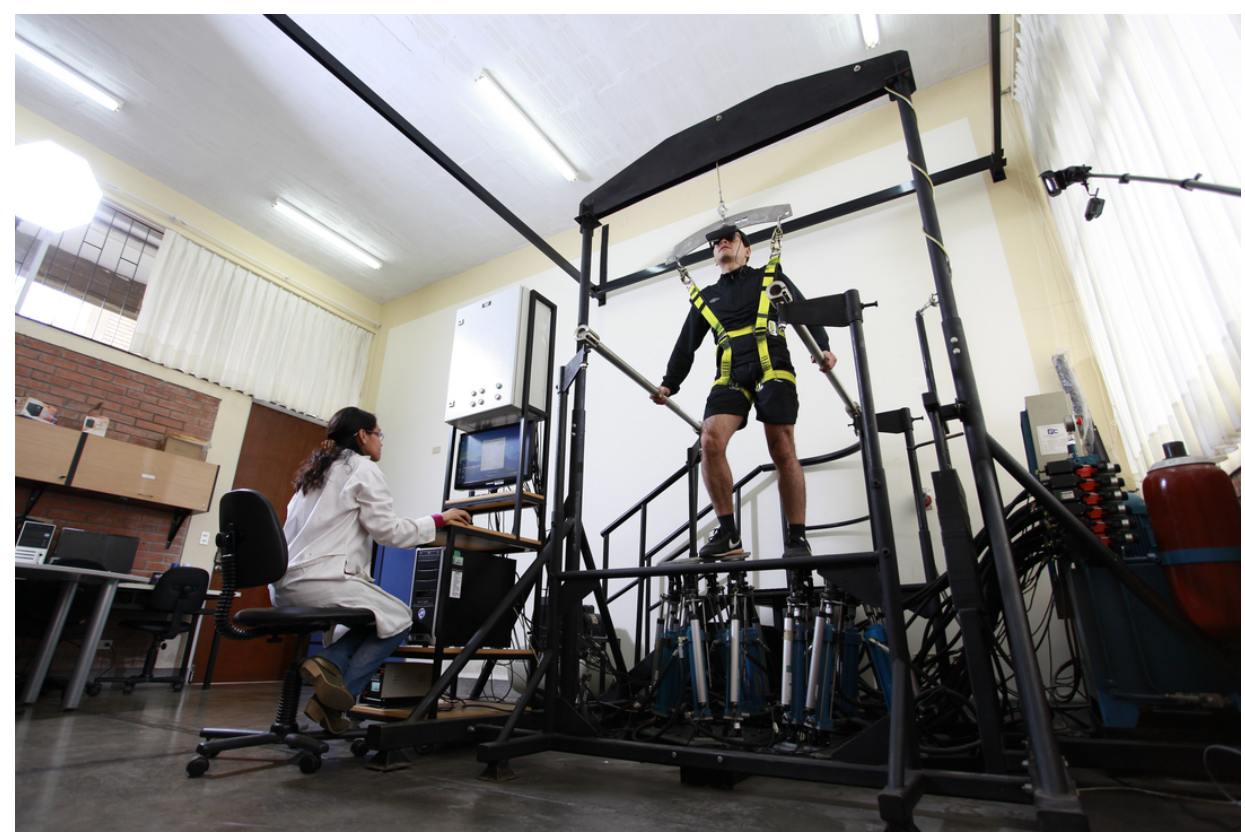

Figure 1. Gait simulator based on two Stewart-Gough platforms. During therapy, a user stands on both platforms, while a therapist supervises the system operation. Several safety measures have been implemented to protect the patient. A schematic describing the elements presented in this figure is provided in Figure 2.

An overview of the gait simulator is illustrated in Figure 2. It is composed of two Stewart-Gough Platforms (SGP), each comprised of six pistons, whose coordinated displacement moves the upper platform to the desired position. They are connected to the Oil Hydraulic Power Unit (OHPU), which is comprised of valves that regulate oil flow through hosepipes in order to increase or decrease pressure and thus produce piston displacement.

The basic design of the SGP is inspired by kinematic and dynamic features of human walking. Thus, one fundamental condition concerns the platform displacement range on the sagittal plane, which must allow adequate step width. The workspace and the maximum output forces produced by the system are listed in Table 1. Regarding the actuators, hydraulic actuation composed of 12 pistons ( 6 for each platform) driven by a $3 \mathrm{~kW}$ power OHPU are controlled by proportional electronic valves. A linear potentiometer is attached to each piston to assess the actual displacement.

Regarding the hardware/software architecture, three computational units allow the user (i.e., therapist) to control the gait simulator. First, the Base Station Unit (BSU) is composed of a "local" Personal Computer (PC) with a Graphical User Interface (GUI) application, which serves to set up the platform operation mode, and an internet-based communication software that establishes communication with a "remote" PC or Remote Unit (RU) for tele-rehabilitation purposes. Second, the Wearable Sensing Unit (WSU) is based on wireless inertial sensors (3space, Yost Technology, Ohio, USA) that allow the acquisition of the patient or therapist movement. Third, the microcontroller-based Control Unit (CU) implements a local closed-loop control of piston displacement. Either the BSU or the RU (i.e., local or remote PCs) may be used to acquired movement data from wireless inertial sensors. 
Table 1. Gait simulator workspace and output forces [20].

\begin{tabular}{cc}
\hline Feature & Maximum Values \\
\hline Static load, $z$ axis & $700 \mathrm{~N}$ \\
Static load, $x$ axis & $200 \mathrm{~N}$ \\
Static load, $y$ axis & $100 \mathrm{~N}$ \\
Linear displacement, $z$ axis & $\pm 150 \mathrm{~mm}$ \\
Linear displacement, $x$ axis & $\pm 400 \mathrm{~mm}$ \\
Linear displacement, $y$ axis & $\pm 50 \mathrm{~mm}$ \\
Rotation, $z$ axis & $\pm 5^{\circ}$ \\
Rotation, $x$ axis & $\pm 5^{\circ}$ \\
Rotation, $y$ axis & $\pm 30^{\circ}$ \\
Linear velocity, $x$ axis & $\pm 1834 \mathrm{~mm} / \mathrm{s}$ \\
Angular velocity, $y$ axis & $\pm 3.5 \mathrm{rad} / \mathrm{s}$ \\
\hline
\end{tabular}



Figure 2. Schematic diagram illustrating the gait simulator composing units, including a representation of main coordinate systems $G_{b}$ (fixed base) and $G_{p}$ (mobile platform).

In order to evaluate the system tele-rehabilitation capabilities between users in different locations, possibly distinct countries, a specific software was developed. In particular, an internet-based software enables the interconnection between the BSU and a RU using TCP/IP connectivity. Through that connection, a therapist may, for instance, obtain realtime information from ongoing therapy, as well as send direct commands to control the robotic device. In this case, time delays and packet loss involved in internet communication may lead to instability. This issue is particularly important, since communication tests were done between Montpellier, France; Brasilia, Brazil; and the gait simulator located in Lima, Perú.

Each parallel manipulator composing the system, referred to as left $l$ and right $r$, has one fixed base and one mobile platform onto which the user stands. The coordinate systems for the fixed base and the mobile platform are defined as $G_{b}$ and $G_{p}$, respectively, as depicted in Figure 2, and the $G_{p}$ origin coincides with the foot median position. From a 
kinematic control perspective, high-level platform motion commands computed by each of the control strategies proposed in this work must enable computing corresponding piston displacements that will provide an appropriate $G_{p}$ trajectory. However, solving the inverse kinematic equations for SGP is complex, due to closed kinematic chain and constrained configuration. In this work, a numerical solution based on unique geometric features of SGP is used. Further details are provided in [14]. For all control strategies, hard-coded measures to avoid inter-platform collision are implemented by reducing the available operational space.

\subsection{Control Strategies}

The gait simulator described in Section 2.1 enables independent 6-DOF movement of each foot. Based on this system, feet trajectories that promote rehabilitation may be created. This basic control strategy is referred to as Fixed Gait Trajectory Mode (FGTM) (Figure 3a). In this work, novel strategies that enable modulating platform movement in real time are proposed.

Regarding the FGTM, emulating standard gait trajectories may be one of the first approaches applied in lower limb robotic rehabilitation systems [2]. For that purpose, feet pose during gait was recorded using optic motion capture [20] to serve as a high-level reference to the $\mathrm{CU}$. The reference trajectory for each foot was measured with respect to the standard double support position. This is the same starting position used in the gait simulator, defined by the rotation matrix ${ }^{b} \mathbf{R}_{p 0}$ and position ${ }^{b} \mathbf{t}_{p 0}$, both known constants in our setup. Note that, in the convention used here, while ${ }^{b} \mathbf{t}_{p 0}$ represents the position of the coordinate system $G_{p 0}$ in terms of $G_{b},{ }^{b} \mathbf{R}_{p 0}$ is used to compute rotations from $G_{p 0}$ to $G_{b}$. For each data point measured by the motion capture system, corresponding high-level commands to the robotic system were computed using Equation (1).

$$
\begin{aligned}
{ }^{b} \mathbf{R}_{p} & ={ }^{b} \mathbf{R}_{p 0}{ }^{p 0} \mathbf{R}_{p} \\
{ }^{b} \mathbf{t}_{p} & ={ }^{b} \mathbf{t}_{p 0}+{ }^{b} \mathbf{R}_{p 0}{ }^{p 0} \mathbf{t}_{p} .
\end{aligned}
$$

\subsubsection{Therapist Modulation Mode (TMM)}

An alternative to the FGTM, in which the robotic motion is performed without any online modulation, is to enable therapists to transfer movements in real-time to the gait simulator. In addition to the possibility of creating arbitrary lower extremity motion, the method is particularly useful for tele-rehabilitation.

In order to track the therapist's feet trajectory, the adopted approach involves the use of sensors, such as wireless inertial sensors, attached to the feet. Other sensing systems may be used, particularly if reliable position tracking is desired. As illustrated in Figure 3b, based on the measured pose with respect to the initial position provided by such sensors, i.e., ${ }^{p 0} \mathbf{R}_{p}$ and ${ }^{p 0} \mathbf{t}_{p}$, the same computations described in Equation (1) may be used to provide high-level commands to the platforms. The main difference with the previous strategy is that those estimates are transferred in real time to the robot.

In tele-rehabilitation, the corresponding time delay may produce instability. This is especially the case when therapy involves fast movements. In order to limit the effects of these potential issues, in addition to optimization of communication software to reduce delay and packet loss and use of low-pass filtering to minimize the effect of high-frequency fluctuations, control gains are reduced. Finally, two additional functionalities are proposed to improve the overall usability of this operation mode. First, the therapist may directly deactivate any DOF or reset his/her initial position at any moment. Moreover, the performed trajectories may be recorded to be used in open-loop, such as in FGTM. 

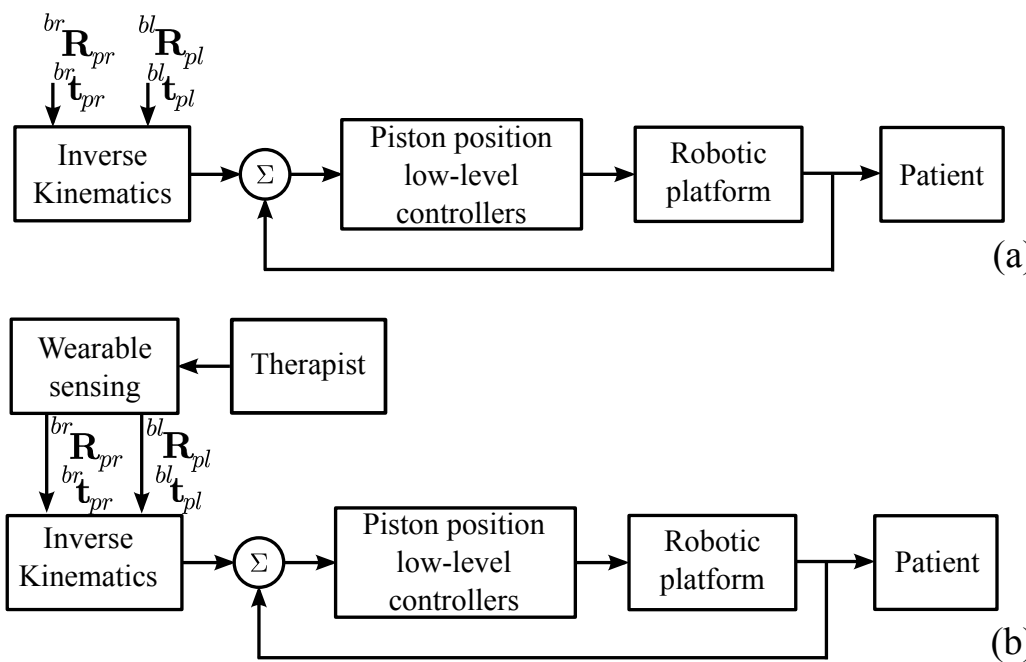

(b)

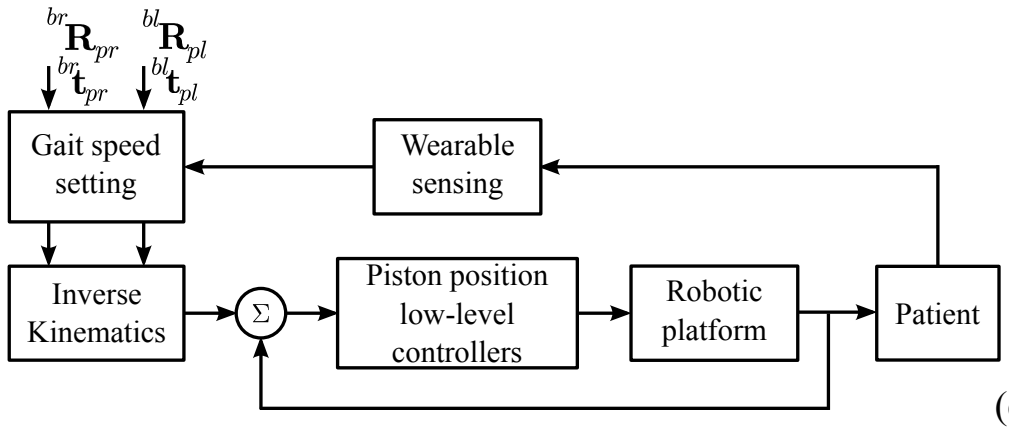

(c)

Figure 3. Control strategies for gait rehabilitation. (a) Represents the basic control strategy using fixed gait trajectories, and $(\mathbf{b}, \mathbf{c})$ depict the novel approaches described in this work, i.e., Therapist Modulation Mode (TMM) and Self-Modulation Mode (SMM), respectively.

\subsubsection{Self-Modulation Mode (SMM)}

In order to increase patient engagement during therapy and consequently the overall positive outcome, an additional proposed control strategy involves real-time modulation of robot motion based on patient control. As a first implementation towards more complex scenarios, discussions with clinicians supported the use of trunk inclination to modulate gait trajectory speed.

For that purpose, a wireless inertial sensor is attached to the patient's upper body. Measurements of upper body angular displacement on the sagittal plane are used to increase or decrease the cadence of gait trajectory employed at the FGTM. Considering an upper-body angle $\theta$, we defined a maximum trunk flexion $\theta_{\max }$, which is used to compute the applied gait speed using Equation (2):

$$
g(\theta)=g_{\alpha} \frac{\theta}{\theta_{\max }}+g_{\min }
$$

where $g$ is the gait speed, $g_{\min }$ is the minimum gait speed $(0 \mathrm{~km} / \mathrm{h}$ in this case, but often $0.8 \mathrm{~km} / \mathrm{h}$ may be used [4]), and $g_{\alpha}$ is a gain defined by the user. In this work, $g_{\alpha}$ is computed considering maximum trunk flexion $\theta_{\max }\left(30^{\circ}\right.$ in this work) leads to the maximum defined gait speed $(4.8 \mathrm{~km} / \mathrm{h}$ in this case).

\subsection{Feasibility Study}

The experiments were designed to investigate the feasibility of both proposed control strategies in terms of system performance and user subjective perception. In this section, data obtained from an individual with no gait impairments (male, 27 years old, $65 \mathrm{~kg}$ ) is depicted. In the trial involving a therapist, another individual took part in the test (male, 34 years old, $77 \mathrm{~kg}$ ). 
Within the experiments performed in this work, the sensors were attached either to the user trunk, where it provided upper-body flexion/extension estimates for the $\mathrm{SMM}$, or to the feet, providing plantar flexion/dorsiflexion, eversion/inversion, and external/internal rotation readings for the TMM. For the latter, ideally foot position would also have to be measured and employed for controlling the SGP, but in this work only attitude was evaluated.

Regarding the protocol, the therapist in the TMM trial and the patient in the SMM trial were told to move the orientation of their feet/trunk freely. In the TMM trial, the therapist was positioned behind the patient, as illustrated in the bottom panel of Figure 4. Although a human subject standing on the platform was not required to evaluate the system performance, we have chosen this configuration to prevent prediction of platform movement by the patient, which would possibly occur in tele-rehabilitation.

Tele-rehabilitation feature is validated by testing interconnection between the BSU (Lima, Peru) and an RU located at Montepellier, France. The BSU and RU have a client and server TCP/IP application, respectively, which allow sending commands to the SGP and requesting data from wireless inertial sensors. Public IPs are assigned to both BSU and RU computers. An initial testing using the "ping" command is conducted to assess the typical delay transmitting and receiving data packets during the experiment. No virtual private network nor dedicated internet connection is used in this experiment. Once the sanity check is done, a TCP/IP port is open in both computers to establish communication. Command transfer and SGP data queries were conducted between 5:00 to 7:00 pm, Montpellier local time, to evaluate the performance requirements during peak hours.

After the experiments, the simulated participant filled a questionnaire to evaluate his experience. Since no standard instrument to evaluate the usability of such a type of system was found, a customized form composed of open questions was applied.

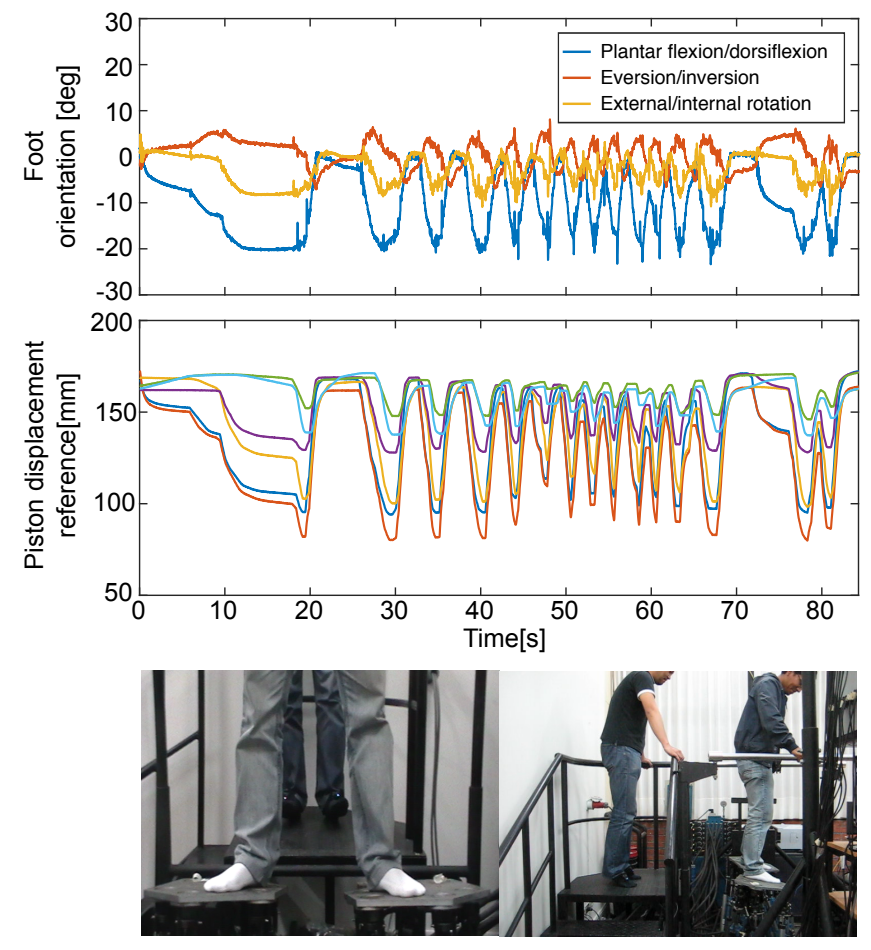

Figure 4. Experimental data from an TMM trial. Top panel shows data from the sensors, which are used to compute the piston displacement reference shown in the center panel. Bottom panel shows two figures that serve to illustrate two distinct instants from the trial. The therapist, using the wireless inertial sensors, input desired set of movements to the mechanical system that are replicated and transmitted to the patient (over the SGP). The tele-rehabilitation feature of the system allows the therapist to conduct this procedure from a remote location. 


\section{Results}

Within the feasibility study described in Section 2.3, series of trials were conducted to explore the system performance, particularly with respect to overall system behavior, and both participants' perceptions as patient and therapist. The ability to be applied in tele-rehabilitation settings was also evaluated.

In Figure 4, the results from the TMM trial are depicted. The data in the first and second row correspond to the right foot orientation and the displacement reference given to the six pistons of the right platform, respectively. Furthermore, the figures in the third row refer to two particular moments when the therapist is performing dorsiflexion (left picture) or plantar flexion (right picture).

In Figure 5, sample results from the SMM trial are depicted. In this trial, the user has increased his trunk inclination until reaching maximum gait speed, and then reduced back until no robot movement was produced. We can observe that the trunk inclination is directly translated into the normalized gait speed (top), and also how this modulation affects the normalized stride duration (bottom).

Regarding tele-rehabilitation validation, inter-connectivity between BSU and RU through internet was tested used the ping command. The mean time to transmit and receive a TCP/IP package (56 data bytes) between RU and BSU (Lima-Montpellier) is $\approx 206$ milliseconds.

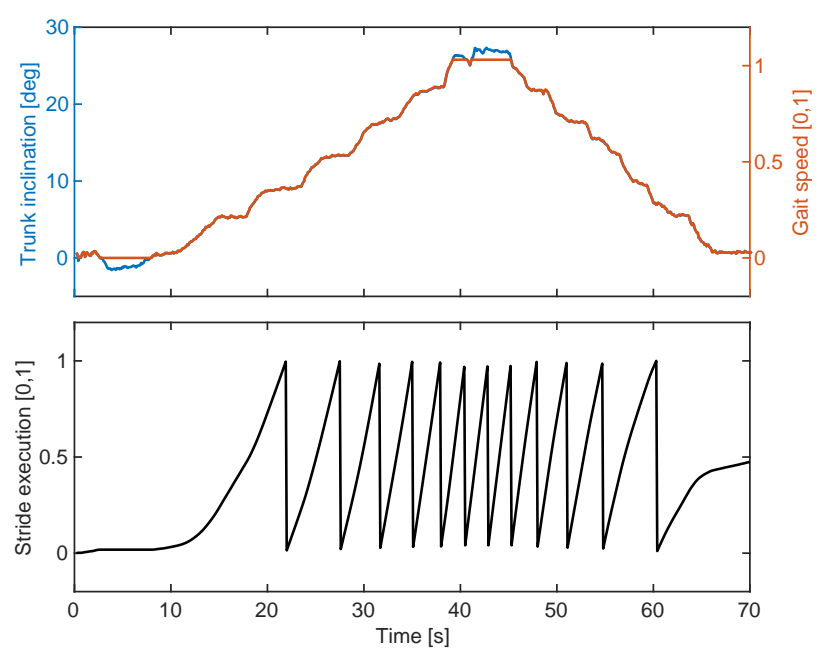

Figure 5. Experimental data from an SMM trial. Top panel shows in blue the trunk inclination in degrees acquired by a wireless inertial sensor during a 70-s experiment. The red line depicts the simultaneous gait speed in $\mathrm{km} / \mathrm{h}$, calculated using Equation (2). Bottom panel depicts the stride execution, i.e., the relative phase of movement of the mobile platform when executing one stride.

\section{Discussion}

To the best of the authors' knowledge, this paper presents a first attempt to implement control strategies such as SMM and TMM in an SGP-based lower limb robot for telerehabilitation. Nevertheless, while the proof-of-concept experiments helped us to evaluate the overall usability of the system, some of its potential limitations became evident.

Within the SMM trial, the user needed to maintain his balance, while simultaneously changing his trunk inclination to modulate gait speed. Despite the supplementary action required in this case, user acceptance was immediate, and no specific concerns were raised. The provided control interface was sufficiently intuitive for the user to control gait speed, as illustrated in Figure 5.

Nonetheless, it may be also noted that other movement intention sources could be used to modulate gait speed, such as myoelectric activity of lower limb muscles, upper limb oscillatory movement, and others. While each of those measures feature specific drawbacks, the strategy employed in this work (i.e., trunk inclination) also present disadvantages. For instance, momentary loss of balance could be wrongly interpreted as 
intention to accelerate or decelerate. While additional signal processing may assist in extracting the volitional movement, we believe further clinical study is required to validate this hypothesis. Furthermore, sensor location in this configuration may be less practical if a harness for body weight support is used. In this case, an alternative is to place the sensor on the neck or another body part.

Regarding the TMM trial, the participant that took the role of the patient mentioned that being unaware of future robot movements produced a degree of anxiety. Since we consider it is an undesirable effect in rehabilitation, strategies must be designed to prevent it. One alternative involves a common practice in physiotherapy: verbally explaining each movement before execution.

One of the potential limiting features when using TMM is that there may be the need to scale therapist movements for a patient of different body dimensions. Nevertheless, particularly considering that the system operates at a limited speed, one finding from these preliminary tests is that the therapist adapts their movement based on the observed patient movement, i.e., avoiding the requirement of additional technical solutions. Indeed, this particular adaptation is commonly observed in human-robot interaction [21,22].

The lack of feedback to the therapist of interaction forces involved may be one additional limitation of the proposed framework when using TMM. Indeed, different modalities exist to provide this real-time information to the therapist [23]. While we recognize this issue may limit the range of interventions that may be applied, we also understand visual (and kinematic) feedback alone are sufficient to enable different therapeutic interventions using such a mechatronic system.

The use of position control on both control strategies, instead of techniques based on force control, features additional implications. For instance, strategies based on force control would enable a higher degree of adaptability depending on the interaction forces between the user and the platform. Nevertheless, when comparing to exoskeleton-based systems, an end-effector system using position control would be safer to exoskeletons using a similar approach, particularly in cases of spasticity, for instance.

Certainly, testing of the tele-rehabilitation feature requires a higher number of experiments from different locations. Intercontinental connection implies that only one country is within the peak hours. Further assessment should be done with an RU located in similar geographical longitude as it might reveal additional issues regarding package loss and delay due to a higher concentration of network users. Moreover, we understand future works should be conducted to investigate the stability of such tele-rehabilitation system.

\section{Conclusions}

As robotic systems for lower limb rehabilitation evolve and new features become available, such as potential use in tele-rehabilitation, new control strategies may be used to further increase the functional outcome of therapy. In this work, we have proposed two novel approaches for controlling a gait simulator based on two Stewart-Gough platforms. One technique enables direct control of the robotic platform by the therapist, which is particularly interesting considering the broad operational space of such a robotic system. Another method aims at improving patient engagement, providing the user limited control over the platform. Here, we enabled control of gait speed using the trunk inclination measured using a wireless sensor. Feasibility experiments were conducted with healthy subjects, and the obtained results indicate the system presents sufficient performance to justify broader quantitative studies.

Author Contributions: Conceptualization, A.P.L.B., M.H. and D.E.; methodology, A.P.L.B., M.H., L.C. and G.C.-P.; software, G.C.-P., L.C., A.P.L.B. and M.H.; data curation, A.P.L.B. and M.H.; writing — original draft preparation, A.P.L.B. and L.C.; writing—review and editing, A.P.L.B., L.C., G.C.-P., M.H. and D.E.; funding acquisition, A.P.L.B., M.H. and D.E. All authors have read and agreed to the published version of the manuscript. 
Funding: This research was funded in the context of the STIC-AmSud Program, with funds from INRIA (France), CAPES (Brazil), and CONCYTEC (Peru).

Institutional Review Board Statement: The study was conducted according to the guidelines of the Declaration of Helsinki and approved by the Pontificia Universidad Católica del Perús Research Ethics Committee.

Informed Consent Statement: Informed consent was obtained from all subjects involved in the study.

Data Availability Statement: Not applicable.

Conflicts of Interest: The authors declare no conflict of interest.

Abbreviations
The following abbreviations are used in this manuscript:
$\begin{array}{ll}\text { BSU } & \text { Base Station Unit } \\ \text { BWSTT } & \text { Body-Weight Supported Treadmill Training } \\ \text { CU } & \text { Control Unit } \\ \text { DOF } & \text { Degree-of-Freedom } \\ \text { FGTM } & \text { Fixed Gait Trajectory Mode } \\ \text { OHPU } & \text { Oil Hydraulic Power Unit } \\ \text { RU } & \text { Remote Unit } \\ \text { SGP } & \text { Stewart-Gough Platform } \\ \text { SMM } & \text { Self-Modulation Mode } \\ \text { TMM } & \text { Therapist Modulation Mode } \\ \text { WSU } & \text { Wearable Sensing Unit }\end{array}$

\section{References}

1. Van der Loos, H.M.; Reinkensmeyer, D.J. Rehabilitation and Health Care Robotics. In Springer Handbook of Robotics; Springer: Berlin/Heidelberg, Germany, 2008; pp. 1223-1251.

2. Jezernik, S.; Colombo, G.; Keller, T.; Frueh, H.; Morari, M. Robotic Orthosis Lokomat: A Rehabilitation and Research Tool. Neuromodul. Technol. Neural Interface 2003, 6, 108-115. [CrossRef] [PubMed]

3. Hesse, S.; Uhlenbrock, D.; Werner, C.; Bardeleben, A. A mechanized gait trainer for restoring gait in nonambulatory subjects. Arch. Phys. Med. Rehabil. 2000, 81, 1158-1161. [CrossRef] [PubMed]

4. de Sousa, A.C.C.; Bó, A.P. Simulation studies on hybrid neuroprosthesis control strategies for gait at low speeds. Biomed. Signal Process. Control 2021, 70, 102970. [CrossRef]

5. Schmidt, H.; Hesse, S.; Bernhardt, R.; Krüger, J. HapticWalker-A novel haptic foot device. ACM Trans. Appl. Percept. 2005, 2, 166-180. [CrossRef]

6. Gui, K.; Liu, H.; Zhang, D. A Practical and Adaptive Method to Achieve EMG-Based Torque Estimation for a Robotic Exoskeleton. IEEE/ASME Trans. Mechatronics 2019, 24, 483-494. [CrossRef]

7. Hornby, T.G.; Campbell, D.D.; Kahn, J.H.; Demott, T.; Moore, J.L.; Roth, H.R. Enhanced Gait-Related Improvements After Therapist- Versus Robotic-Assisted Locomotor Training in Subjects With Chronic Stroke: A Randomized Controlled Study. Stroke 2008, 39, 1786-1792. [CrossRef] [PubMed]

8. Kim, J.; Park, H.S.; Damiano, D.L. An Interactive Treadmill Under a Novel Control Scheme for Simulating Overground Walking by Reducing Anomalous Force. IEEE/ASME Trans. Mechatron. 2015, 20, 1491-1496. [CrossRef]

9. Hernandez, E.; Warhmund, C.; Lamoureux, K.; Lee, E.; Sanchez, I.; Matthews, W.; Jafari, A. A Novel Treadmill That Can Bilaterally Adjust the Vertical Surface Stiffness. IEEE/ASME Trans. Mechatron. 2018, 23, 2338-2346. [CrossRef]

10. Girone, M.; Burdea, G.; Bouzit, M.; Popescu, V.; Deutsch, J. A Stewart Platform-Based System for Ankle Telerehabilitation. Auton. Robot. 2001, 10, 203-212. [CrossRef]

11. Forrester, L.W.; Roy, A.; Krywonis, A.; Kehs, G.; Krebs, H.I.; Macko, R.F. Modular ankle robotics training in early subacute stroke: A randomized controlled pilot study. Neurorehabilit. Neural Repair 2014, 28, 678-687. [CrossRef] [PubMed]

12. Li, B.; Williamson, J.; Kelp, N.; Dick, T.; Bo, A.P.L. Towards balance assessment using Openpose. In Proceedings of the 2021 43rd Annual International Conference of the IEEE Engineering in Medicine and Biology Society, Guadalajara, Mexico, 31 October-4 November 2021; pp. 1-4.

13. Yoon, J.; Novandy, B.; Yoon, C.-H.; Park, K.-J. A 6-DOF Gait Rehabilitation Robot With Upper and Lower Limb Connections That Allows Walking Velocity Updates on Various Terrains. IEEE/ASME Trans. Mechatron. 2010, 15, 201-215. [CrossRef]

14. Sevillano, G.; Elias, D.; Callupe, R.; Marcacuzco, R.; Barriga, B. Gait Simulator Based on the Parallel Stewart-Gough Platform. In Interdisciplinary Applications of Kinematics; Springer: Dordrecht, The Netherlands, 2012; pp. 99-108. 
15. Tzafestas, C.; Velanas, S.; Fakiridis, G. Adaptive impedance control in haptic teleoperation to improve transparency under time-delay. In Proceedings of the 2008 IEEE International Conference on Robotics and Automation, Pasadena, CA, USA, 19-23 May 2008; pp. 212-219. [CrossRef]

16. Cortese, M.; Cempini, M.; de Almeida Ribeiro, P.R.; Soekadar, S.R.; Carrozza, M.C.; Vitiello, N. A Mechatronic System for Robot-Mediated Hand Telerehabilitation. IEEE/ASME Trans. Mechatron. 2015, 20, 1753-1764. [CrossRef]

17. Carignan, C.R.; Krebs, H.I. Telerehabilitation robotics: Bright lights, big future? J. Rehabil. Res. Dev. 2006, 43, 695. [CrossRef] [PubMed]

18. de Souza Baptista, R.; Bo, A.P.L.; Hayashibe, M. Automatic Human Movement Assessment With Switching Linear Dynamic System: Motion Segmentation and Motor Performance. IEEE Trans. Neural Syst. Rehabil. Eng. 2017, 25, 628-640. [CrossRef] [PubMed]

19. Bo, A.P.L.; Poignet, P.; Widjaja, F.; Ang, W.T. Online pathological tremor characterization using extended Kalman filtering. In Proceedings of the 2008 30th Annual International Conference of the IEEE Engineering in Medicine and Biology Society, Vancouver, BC, Canada, 20-25 August 2008; pp. 1753-1756.

20. Elias, D.; Callupe, R.; Ceccarelli, M. Characteristics of a Walking Simulator with Parallel Manipulators. In Interdisciplinary Applications of Kinematics; Springer International Publishing: Berlin/Heidelberg, Germany, 2015; pp. 137-145.

21. Marinho, M.M.; Geraldes, A.A.; Bo, A.P.L.; Borges, G.A. Manipulator control based on the dual quaternion framework for intuitive teleoperation using kinect. In Proceedings of the 2012 Brazilian Robotics Symposium and Latin American Robotics Symposium, Fortaleza, Brazil, 16-19 October 2012; pp. 319-324.

22. Adorno, B.V.; Bo, A.P.L.; Fraisse, P. Kinematic modeling and control for human-robot cooperation considering different interaction roles. Robotica 2015, 33, 314-331. [CrossRef]

23. Atashzar, S.F.; Shahbazi, M.; Tavakoli, M.; Patel, R.V. A Computational-Model-Based Study of Supervised Haptics-Enabled Therapist-in-the-Loop Training for Upper-Limb Poststroke Robotic Rehabilitation. IEEE/ASME Trans. Mechatron. 2018, 23, 563-574. [CrossRef] 